\title{
Pressure dependence of the refractive index of monoclinic and yttria-stabilized cubic zirconia
}

\author{
N. M. Balzaretti and J. A. H. da Jornada \\ Instituto de Física, Universidade Federal do Rio Grande do Sul, Porto Alegre, Rio Grande do Sul, \\ Codigo de Enderecamento Postal 91501-970, Brazil
}

(Received 6 February 1995)

\begin{abstract}
The pressure dependence of the refractive index of monoclinic and yttria-stabilized cubic zirconia was measured up to 3 and $8 \mathrm{GPa}$, respectively, using an interferometric method with the diamond-anvil cell. The experimental results show a practically linear behavior for both crystalline structures. For cubic zirconia, the refractive index remains practically constant under pressure, while for the biaxial monoclinic phase, one component of the refractive index decreases and the other component increases with pressure, despite the rather isotropic lattice compression in the corresponding crystalline directions. The behavior of the electronic polarizability of the oxygen ion was investigated through the LorentzLorenz relation, yielding the following Mueller parameters: for the cubic phase $\Lambda_{0}=1.02$, and for the monoclinic phase $\Lambda_{0}=1.05$ for the component nearly parallel to the $b$ direction, and $\Lambda_{0}=0.85$ for the component in the direction of the projection of the " $a$ " axis on the sample plane. In the case of monoclinic zirconia, the behavior of the polarizability under pressure is about $20 \%$ more anisotropic than the compression of the respective lattice parameters, indicating a distinct effect of the interatomic interactions over the oxygen ions for the two directions under consideration.
\end{abstract}

\section{INTRODUCTION}

In the literature there are many theoretical and experimental works concerning several aspects of zirconia $\left(\mathrm{ZrO}_{2}\right)$. From the point of view of applications, $\mathrm{ZrO}_{2}$ is a very important material due to its high refractive index, hardness, and stability in oxidizing atmospheres at high temperatures. Transparent single crystals of stabilized cubic $\mathrm{ZrO}_{2}$ are optical materials of high quality with remarkable ionic and mechanical properties.

The monoclinic phase and the yttria-stabilized cubic $\mathrm{ZrO}_{2}$ are stable at ambient temperature and pressure, and have similar properties concerning the bond distances and coordination numbers. ${ }^{1}$ The crystalline structure of $c-\mathrm{ZrO}_{2}$ stabilized with $\mathrm{Y}_{2} \mathrm{O}_{3}$ is of $\mathrm{CaF}_{2}$ type, where some $\mathrm{Zr}^{+4}$ ions are randomly replaced by $\mathrm{Y}^{+3}$ ions, generating several oxygen vacancies necessary to preserve charge neutrality. The structure of the monoclinic phase is also a distortion of the $\mathrm{CaF}_{2}$ type, where the $\mathrm{Zr}$ atoms have sevenfold coordination.

During the past few years we have carried out a general study of the pressure dependence of the refractive index of several materials such as, for example, $\mathrm{MgO},{ }^{2}$ sapphire, ${ }^{3} c-\mathrm{BN},{ }^{4}$ diamond, cubic $\mathrm{SiC}$, and some fluorides, using an interferometric method with the diamond-anvil cell (DAC). From experimental results, we can obtain information about the microscopic behavior of the electronic polarizability during lattice compression. Zirconia is an interesting candidate for our general study due to its important optical and mechanical properties. Moreover, it would be possible to make a comparison between the results for different crystalline structures of the same compound and, also, to investigate the influence of the asymmetry of the monoclinic system over the behavior of the electronic polarizability.

In this work, experimental results for the pressure dependence of the refractive index are given up to $8 \mathrm{GPa}$ for the cubic yttria-stabilized $\mathrm{ZrO}_{2}\left(20 \mathrm{~mol} \% \mathrm{Y}_{2} \mathrm{O}_{3}\right)$ and up to the phase transition for the monoclinic phase, about $3.7 \mathrm{GPa}^{5}$ The Lorentz-Lorenz classical approach is used to investigate the behavior of the polarizability of the oxygen ion in both structures during volume compression.

\section{EXPERIMENTAL TECHNIQUE}

The pressure dependence of the refractive index was measured using the interferometric method described in details in Ref. 2. Briefly, it consists of following the interference fringes of a microinterferometer (sample) during the pressure variation inside the DAC. A ruby calibrant is used to measure the pressure, and a methanolethanol-water mixture is used as the pressuretransmitting medium. An optical system was especially designed to obtain the transmitted interference spectrum from the sample during the pressure change. From the condition of maximum interference for normal incidence, the wavelength displacement of a given fringe due to volume and refractive index variations is given by

$$
\frac{\Delta \lambda_{m}}{\lambda_{m}(0)}=\frac{n(P)}{n(0)} \frac{l(P)}{l(0)}-1,
$$

where $\lambda_{m}$ is the pressure-dependent wavelength of the fringe of order $m, l$ is the sample thickness, and $n$ is the refractive index.

The cubic sample was polished down to a thickness of about $40 \mu \mathrm{m}$, forming two parallel plane surfaces. Over these surfaces was deposited a semitransparent film of gold. On the other hand, the single crystals of monoclinic zirconia already had the dimensions and parallelism necessary for the observation of the optical interference, and the gold film was just deposited over the two parallel 
surfaces to improve the finesse of the transmitted spectrum.

The monoclinic $\mathrm{ZrO}_{2}$ is a biaxial material so, except for the light incidence in the direction of one of the optical axes, there will be a superposition of interference spectra corresponding to the two different refractive indexes for a given propagation direction. In the present case, the single-crystal plane was perpendicular to the $c$ axis. In order to separate the two fringe patterns, we used a polarizer to analyze the transmitted light in two perpendicular directions: one of them nearly parallel to the $b$ axis; and the other nearly parallel to the projection of the $a$ axis on the sample plane, that is, the component $a \sin \beta .^{6}$

\section{EXPERIMENTAL RESULTS AND DISCUSSION}

The wavelength shift of a given interference fringe during the pressure variation can be used to determine the pressure dependence of the refractive index, using Eq. (1) and an equation of state to take into account the respective thickness variations. We used the Murnaghan equation of state to obtain the volume variation of the cubic phase, with $B_{0}=210.3 \mathrm{GPa}\left(20-\mathrm{mol}_{\%} \mathrm{Y}_{2} \mathrm{O}_{3}\right)$ (Ref. 7) and $B_{0}^{\prime}=4.5$.

For the anisotropic monoclinic phase, the variations of the cell parameters are given by ${ }^{8}$

$$
\begin{aligned}
& a=5.138-0.0150 P, \\
& b=5.214-0.0155 P, \\
& c=5.314-0.0117 P, \\
& \beta=99.09-0.0270 P,
\end{aligned}
$$

where the cell parameters are expressed in $\AA$, $\beta$ in degrees, and $P$ in GPa. In the present case, the thickness change corresponds to the compression of the $c$ parameter.

Figure 1 compares the experimental results for $n(P) / n(0)$ for the two structures. The wavelength region was about $690 \mathrm{~nm}$, and it was considered a small optical

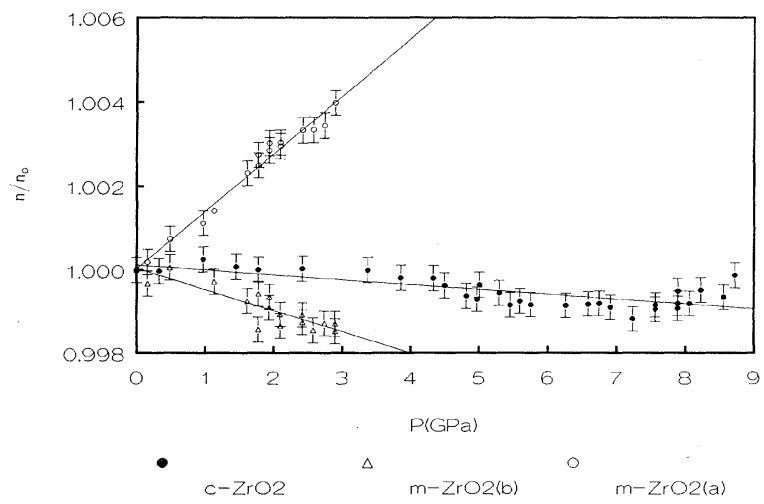

FIG. 1. Pressure dependence of the refractive index of zirconia. (a) and (b) are the components parallel to the crystalline directions $a \sin \beta$ and $b$, respectively. dispersion of the refractive index due to the fringe displacement: $(\partial n / \partial \lambda)=-1.1 \times 10^{-4} \mathrm{~nm}^{-1}$. As can be seen, in the case of the yttria-stabilized $c-\mathrm{ZrO}_{2}$ the refractive index remains practically constant up to $8 \mathrm{GPa}$, while for the monoclinic phase one component slightly decreases and the other increases with pressure. A linear least-square fit yields $(1 / n)(d n / d P)=(-1.0 \pm 0.5)$ $\times 10^{-4} \mathrm{GPa}^{-1}$ for the cubic phase, $(1 / n)(d n / d P)$ $=(-5.0 \pm 0.5) \times 10^{-4} \mathrm{GPa}^{-1}$ for the $b$ component, and $(1 / n)(d n / d P) \quad(+13.5 \pm 0.5) \times 10^{-4} \mathrm{GPa}^{-1}$ for the $a \sin \beta$ component of the monoclinic phase.

It is interesting to analyze these experimental results in the context of the electronic polarizability $(\alpha)$ of the ions. As usual, the polarizability of the oxygen ion, $\alpha\left(O^{-2}\right)=3.88 \times 10^{-3} \mathrm{~nm}^{3}$, is large compared to the polarizability of the positive ion, $\alpha\left(\mathrm{Zr}^{+4}\right)=0.3 \times 10^{-3} \mathrm{~nm}^{3}$. 9 Therefore, only the variation of the oxygen polarizability will be considered during the lattice compression.

While theoretical ab initio studies of the electronic polarizability are complicated, the classical approach of Lorentz-Lorenz provides a simple relationship among refractive index, density $(\rho)$, and polarizability:

$$
\frac{n^{2}-1}{n^{2}+2}=\frac{4 \pi}{3} \rho \alpha \text {. }
$$

Though the $4 \pi / 3$ factor is valid only for cubic systems, this relation will also be used in the case for the monoclinic phase, since we will determine only the relative volume variation of $\alpha$. In Fig. 2 we can see the volume dependence of the electronic polarizability for the cubic and monoclinic phases obtained with Eq. (3), the experimental values for $n(P) / n(0)$, and the Murnaghan equation of state. In the case of the monoclinic phase, we used $B_{0}=112 \mathrm{GPa}$ and $B_{0}^{\prime}=5$ (Ref. 10) to estimate the density variation with pressure.

The volume dependence of $\alpha$ is generally represented by the Mueller parameter ${ }^{11}$ defined as

$$
\Lambda_{o}=\frac{\partial \ln \alpha}{\partial \ln V} \text {. }
$$

The constant values obtained are $\Lambda_{0}=1.02 \pm 0.01$, for the

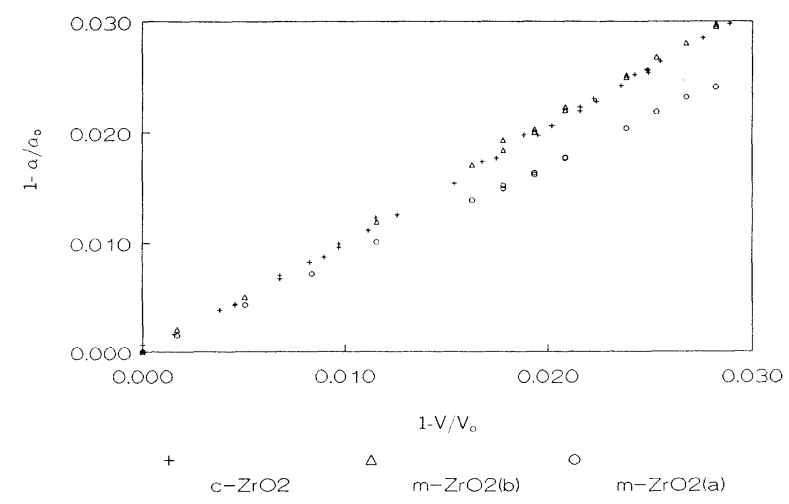

FIG. 2. Volume dependence of the electronic polarizability of zirconia. 
cubic phase, and $\Lambda_{0}=1.05 \pm 0.01$ for the monoclinic phase, with the polarizer direction nearly parallel to the $b$ axis and $\Lambda_{0}=0.85 \pm 0.01$ with the polarizer direction nearly parallel to the component $a \sin \beta$. A value of $\Lambda_{0}$ close to unity means that the relative decreases of $\alpha$ and $V$ are practically the same, and consequently, the refractive index will remain nearly constant under pressure. This seems to be the situation for the cubic $\mathrm{ZrO}_{2}$, and for the component parallel to the $b$ axis in the case of the monoclinic $\mathrm{ZrO}_{2}$. For the component parallel to $a \sin \beta$, $\Lambda_{0}$ is smaller than 1, meaning that the decrease of the electronic polarizability of the oxygen ion in this direction is slower than the corresponding volume compression. As a result, the refractive index increases with pressure.

For the monoclinic phase, the refractive indexes are different for both directions under consideration: $n=2.243$ in the $a \sin \beta$ direction and $n=2.236$ in the $b$ direction. ${ }^{12}$ The correspondent asymmetry of the oxygen polarization at ambient pressure will, according to Eq. (3), be $\alpha_{a \sin \beta} / \alpha_{b}=1.003$. The effect of volume compression was to increase this asymmetry significantly since

$$
\frac{\Delta \alpha_{a \sin \beta} / \alpha_{a \sin \beta}}{\Delta \alpha_{b} / \alpha_{b}}=0.8
$$

for the pressure range up to $3 \mathrm{GPa}$. On the other hand, the correspondent variations of the respective cell parameters are practically the same:

$$
\frac{\Delta(a \sin \beta) /(a \sin \beta)}{\Delta b / b}=0.97 \text {. }
$$

Therefore, the increase of the asymmetry of the electronic polarizability with pressure cannot be totally explained by the small lattice anisotropic compression. This interesting result should be related to short-range interactions between oxygen ions.

The layer-type structure of the monoclinic phase consists of layers of triangular coordination polyhedra of $\mathrm{O}_{\mathrm{I}} \mathrm{Zr}_{3}$ and distorted tetrahedra of $\mathrm{O}_{\mathrm{II}} \mathrm{Zr}_{4} \cdot{ }^{13}$ The $\mathrm{Zr}$ cations are in layers parallel to the (100) plane, in between the layers of $\mathrm{O}_{\mathrm{II}}$ and $\mathrm{O}_{\mathrm{I}}$ ions. ${ }^{14}$ The $a \sin \beta$ component nearly corresponds to the distance between parallel planes of zirconium cations and oxygen anions, i.e., it is an interlayer distance, while the $b$ parameter corresponds to the intralayer distance, as shown in Fig. 2 of Ref. 13. As a result, the short-range repulsive interactions between the oxygen ions in the $b$ direction will be greater than in the $a \sin \beta$ direction. In fact, as the pressure increases, the electronic polarizability decreases faster in the $b$ direction than in the $a \sin \beta$ direction, increasing the asymmetry of $\alpha$.

Shanker and Agarwal ${ }^{15}$ proposed a cubic relation between the polarizability and the ionic radius of the form $\alpha=k r^{3}$, where $k$ is a constant. To obtain an anisotropic electronic polarizability it is necessary to consider an el- lipsoidal form for the oxygen ion, with different radii for the two directions under consideration. We suggest the following relationship to estimate the relative variation of the dimensions of the anion during the pressure variation:

$$
\left(\frac{\alpha}{\alpha_{0}}\right]_{x}=\left[\frac{V}{V_{0}}\right)^{\Lambda_{o x}}=\left[\frac{r}{r_{0}}\right]_{x}^{3},
$$

where $x$ corresponds to $b$ or $a \sin \beta$. Accordingly, the decrease of the radius in the $b$ direction would be $20 \%$ greater than the decrease of the radius in the $a \sin \beta$ direction, while the correspondent decrease of the cell parameters differ only by about $3 \%$.

\section{CONCLUSIONS}

The pressure dependence of the refractive index of cubic and monoclinic $\mathrm{ZrO}_{2}$ was measured using an interferometric method. For the monoclinic phase, the component of the refractive index almost parallel to the layers of oxygen ions slightly decreases with pressure, and the component perpendicular to the layers increases with pressure, while for the cubic phase the refractive index has an intermediate behavior, remaining practically constant under pressure.

For the monoclinic phase, the anisotropy of the electronic polarizability of the oxygen ion increases by about $20 \%$ during the volume compression, despite the small anisotropy of the lattice compression, which is only about $3 \%$. This significant asymmetry of the polarizability behavior should be related to the distinct environments for the oxygens ions in both directions under consideration: in the $b$ direction, an oxygen ion is surrounded by other oxygen ions, and the short-range repulsive interactions should play an important role; in the $a \sin \beta$ direction, on the other hand, the oxygen ions are intercalated with zirconium ions, reducing the effect of the anionanion repulsive interactions. Consequently, the polarizability should decrease faster in the first case, according to the experimental results.

Comparing the cubic and monoclinic zirconia it is possible to say that the behavior of the electronic polarizability of the oxygen ion under pressure is different for different crystalline structures of the same compound. This should be the case, since we are dealing with different interatomic distances and ion environment, and the electronic polarizability seems to depend strongly on the short-range repulsive interactions.

\section{ACKNOWLEDGMENTS}

We are deeply grateful to A. S. Pereira (Esc.Engenharia-UFRGS-Brasil) for helpful discussions about the present work. This research is partially supported by CNPq, FAPERGS, and FINEP.

${ }^{1}$ P. Li, I. Chen, and J. E. Penner-Hahn, Phys. Rev. B 48, 10063 (1993).

${ }^{2}$ N. M. Balzaretti and J. A. H. da Jornada, High Pressure Res. 2, 183 (1990).

${ }^{3}$ N. M. Balzaretti, J. P. Denis, and J. A. H. da Jornada, J. Appl. Phys. 73, 1426 (1993).

${ }^{4}$ N. M. Balzaretti and J. A. H. da Jornada, in Proc. XXXII Annual Meeting of the European High Pressure Research Group, 
29th Aug.-1st Sept. 1994, edited by J. Kamarad, Z. Arnold, and A. Kapicka (Technical University Brno, Czech Republic, 1994), pp. 125-129.

${ }^{5}$ A. S. Pereira and J. A. H. da Jornada, J. Mater. Sci. Lett. 8, 1353 (1989).

${ }^{6}$ A. S. Pereira and J. A. H. da Jornada, J. Mater. Res. 9, 1059 (1994).

${ }^{7}$ D. W. Liu, C. H. Perry, A. A. Feinberg, and R. Currat, Phys. Rev. B 36, 9212 (1987).

${ }^{8}$ J. M. Leger, P. E. Tomazewski, A. Atouf, and A. S. Pereira, Phys. Rev. B 47, 14075 (1993).

${ }^{9}$ L. Pauling, Proc. R. Soc. London Ser. A 114, 181 (1927).

${ }^{10}$ A. S. Pereira, J. M. Leger, and J. A. H. da Jornada, Acta
Metall. Mater. 42, 2701 (1994).

${ }^{11}$ H. Mueller, Phys. Rev. 47, 947 (1935).

${ }^{12}$ W. E. Tröger, Optical Determination of Rock-Forming Minerals, 4th ed., edited by H. U. Bambauer, F. Taborszky, and H. D. Trochin (E. Schweizerbart'sche Verlagsbuchhandlung, Stuttgart, 1979), Pt. I.

13J. D. McCullough and K. N. Trueblood, Acta Crystallogr. 12, 507 (1959).

${ }^{14}$ E. C. Subbarao, H. S. Maiti, and K. K. Srivastava, Phys. Status Solidi A 21, 9 (1974).

15J. Shanker and S. K. Agarwal, J. Phys. Chem. Solids 37, 443 (1976). 